\title{
Human Papillomavirus Related Multiphenotypic Sinonasal Carcinoma: Report of a Case with Early and Progressive Metastatic Disease
}

\author{
Alejandro I. Rodarte ${ }^{1,2}$ Anuraag S. Parikh ${ }^{1,2}$ Shekhar K. Gadkaree ${ }^{1,2}$ Ashton E. Lehmann ${ }^{1,2}$ \\ William C. Faquin ${ }^{1,2}$ Eric H. Holbrook ${ }^{1,2}$ Derrick T. Lin ${ }^{1,2}$
}

\footnotetext{
1 Department of Otolaryngology - Head and Neck Surgery, Massachusetts Eye and Ear, Boston, Massachusetts, United States

2 Department of Otolaryngology, Harvard Medical School, Boston, Massachusetts, United States
}

\begin{abstract}
Address for correspondence Anuraag S. Parikh, MD, Department of Otolaryngology - Head and Neck Surgery, Massachusetts Eye and Ear, 243 Charles Street, Boston, MA 02114, United States (e-mail: anuraag_parikh@meei.harvard.edu).
\end{abstract}

J Neurol Surg Rep 2019;80:e41-e43.

\begin{abstract}
Keywords

- human papillomavirus related multiphenotypic sinonasal carcinoma

- HPV type 16

- sinonasal carcinoma

- adenoid cystic carcinoma

- HPV-related carcinoma with adenoid cysticlike features

Introduction Human papillomavirus (HPV) related multiphenotypic sinonasal carcinoma (HMSC) is a newly described entity. The prognosis of this neoplasm is not well understood, but cases often demonstrate high-grade features and paradoxically indolent behavior.

Case Report We present the case of a 65-year-old man referred with unilateral nasal congestion and epistaxis. Nasal endoscopy and imaging revealed an expansile mass filling the left nasal cavity. The patient underwent endoscopic-assisted medial maxillectomy, with complete resection and negative margins. Pathology revealed a basaloid tumor consisting of solid nests with multiple foci of cribriform architecture, with positivity for high-risk HPV, thus supporting the diagnosis of HMSC. Postoperatively, the patient received 66.6-Gy adjuvant three-dimensional proton therapy. Twenty-three months after surgical resection, he developed enlarging pulmonary nodules with biopsy features consistent with the primary sinonasal tumor. He was treated with three cycles of chemotherapy and eight cycles of immunotherapy, progressing on both therapies. He remains on palliative chemotherapy.

Conclusion Here, we present a case of HMSC with early and progressive distant metastasis. We aim to add to an understanding of the behavior of this entity. Although this neoplasm may typically be indolent, further classification of high-risk features is necessary to identify rare aggressive cases.
\end{abstract}

\section{Introduction}

Malignant neoplasms of the sinonasal tract are uncommon. These tumors are diverse and include a variety of histopathologies such as squamous cell carcinoma (SCC) and adenoid cystic carcinoma (ACC). ${ }^{1}$ Human papillomavirus (HPV) related multiphenotypic sinonasal carcinoma (HMSC), also known as HPV-related carcinoma with adenoid cystic-like features, is a recently described neoplasm restricted to the sinonasal tract,

with histological features of high-grade, solid-type ACC, with HPV positivity and paradoxically indolent behavior. ${ }^{2-4}$

HMSC was provisionally included in the 2017 World Health Organization classification of head and neck tumors as a candidate to be a separate tumor entity, different from ACC and SCC. ${ }^{5}$ In contrast to ACC, HMSC (1) is associated with HPV, most commonly type 33, (2) may have overlying squamous dysplasia/SCC of the surface epithelium, (3) fails
License terms

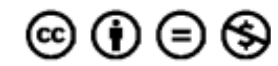

received

May 16, 2019

accepted

May 25, 2019
DOI https://doi.org/

10.1055/s-0039-3399571. ISSN 2193-6358. (c) 2019 Georg Thieme Verlag KG
Stuttgart · New York 
to demonstrate the MYB gene fusions, and (4) has a more indolent clinical behavior., ${ }^{3,6}$

The largest case series of HMSC is described by Bishop et al, who first suggested that these tumors have a favorable outcome. Most reports published since this series have followed this trend. ${ }^{1,3,4,6-10}$ To our knowledge, only a few recorded cases have documented locally aggressive tumors or very late distant metastasis, and there have been no reported tumor-related deaths. ${ }^{3,11,12}$ Here, we present a case of HMSC with early and progressive metastatic disease. With this report, we aim to add to the understanding of the behavior of this new clinical entity.

\section{Case Report}

A 65-year-old man was referred to the Department of Otolaryngology with unilateral nasal congestion and epistaxis. Anterior rhinoscopy revealed a large mass occupying the entire left nasal cavity. The tumor was biopsied and initially diagnosed as nonkeratinizing HPV-related SCC. The patient underwent an endoscopic-assisted medial maxillectomy, achieving a gross total resection with negative margins.

Pathology revealed a basaloid tumor consisting of solid nests with multiple foci of cribriform architecture (-Fig. $\mathbf{1}$ ). There was no overt squamous differentiation identified within the tumor, but surface epithelial squamous dysplasia was present. HPV testing by real-time polymerase chain reaction (Roche cobas 4800 HR-HPV analysis, Roche, Basel, Switzerland) was positive for HPV-16 and was confirmed by HPV-16/18 RNA in situ hybridization (RNAscope ACD HR16/18, Advanced Cell Diagnostics Inc., Newark, California, United States), showing positive staining within the carcinoma (-Fig.2), thus supporting the diagnosis of HMSC. Immunohistochemical staining showed diffuse positivity for $\mathrm{p} 16$. In addition, the carcinoma was positive for p63 and CK7 and showed focal positivity for S100, calponin, and smooth muscle actin (-Fig. 2).

Two months postoperatively, the patient underwent threedimensional proton therapy to the paranasal sinuses to a total dose of 66.6 Gy. Twenty-three months after the surgical resection, he developed enlarging pulmonary nodules that had histological features consistent with metastasis from the primary sinonasal tumor. He was treated with three cycles of chemotherapy (carboplatin/taxol), with initial partial response but subsequent progression. He was then treated with immunotherapy (pembrolizumab) for eight cycles, but it was discontinued because of evidence of progressive disease on the PET-CT (positron emission tomography-computed tomog-

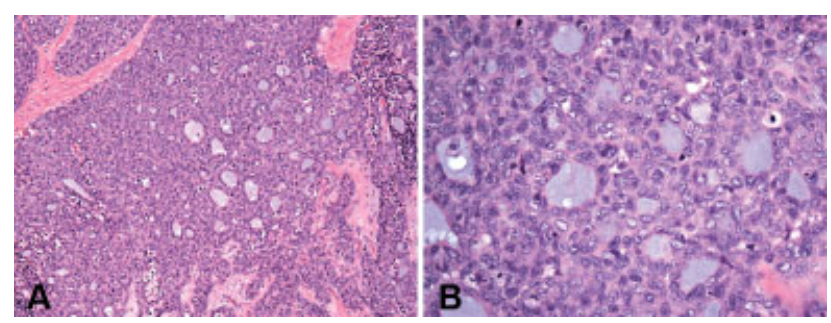

Fig. 1 Hematoxylin and eosin staining at (A) low and (B) high power showing solid nests with multiple foci of cribriform architecture.

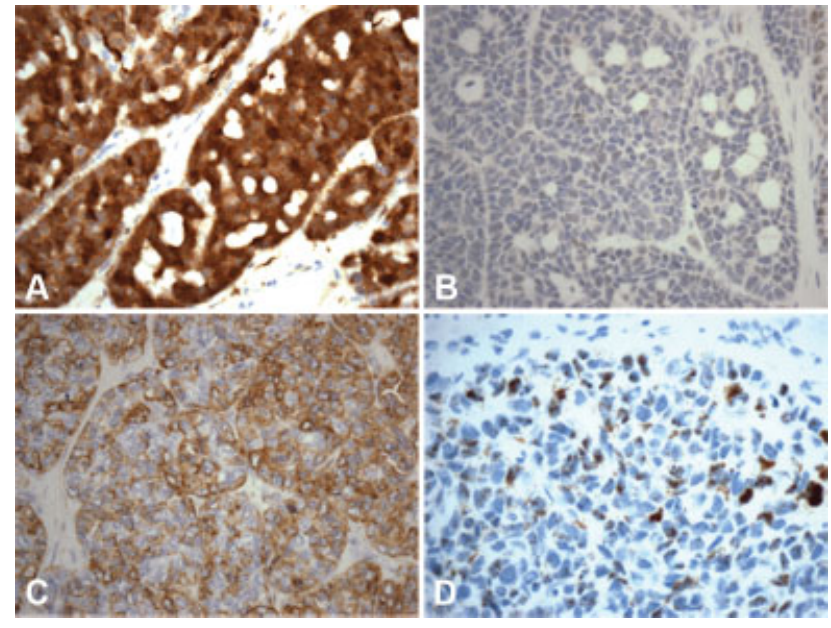

Fig. 2 On immunohistochemistry stainings, the tumor was positive for (A) p16 and displayed focal positivity for (B) MYB. (C) Smooth muscle actin, along with other myoepithelial markers, was focally positive. (D) RNA in situ hybridization was positive for high-risk human papillomavirus within the tumor.

raphy). He remains on palliative chemotherapy (fluorouracil/ cetuximab).

\section{Discussion}

HMSC is a newly identified pathological entity that has been suggested to be indolent despite a high-grade appearance on histopathology. Given the limited number of reported cases, the clinical behavior and consensus treatment guidelines have not yet been established. However, in view of the typically indolent behavior, it has been suggested that surgery with close monitoring has resulted in a good prognosis. ${ }^{1,9}$ This management is in direct contrast with most sinonasal malignancies that are treated with adjuvant therapy. Our case, however, is one with early and progressive metastatic disease despite gross total resection and adjuvant radiation therapy, highlighting an aggressive subset of this disease and the need to properly stratify patients to determine the need for adjuvant radiation or chemoradiation therapy.

Most HMSC cases reported have positive outcomes following surgical removal alone or accompanied by radiotherapy. In the limited cases that recur, the tumors are slow growing, with the timing of recurrence ranging widely: the earliest local recurrence previously reported occurred 23 months posttreatment, and the latest recurrence previously reported occurred after a 30-year disease-free interval. ${ }^{1,3,4,6-11}$ Most previously reported recurrences responded well to treatment, achieving long disease-free intervals. Only two cases previously reported progressed to distal metastasis after 96 and 144 months following their initial treatment. ${ }^{3}$ Our case differs from others reported in the literature due to the very early development of distal metastasis in less than 2 years following the initial treatment. Furthermore, our patient's recurrence progressed even after chemotherapy and immunotherapy. We were not able to find reports of another HMSC tumor with such aggressive behavior, leading us to speculate that there may be a relation between its odd clinical and molecular characteristics. 
In the past, HMSC cases have frequently carried the diagnosis of ACC. As previously mentioned, several characteristics differentiate HMSC from ACC. Yet, another important diagnostic entity in the differential is basaloid SCC. Morphologically, it is difficult to distinguish HMSC from ACC or basaloid SCC due to the presence of solid and cribriform architecture and highly cellular lesions composed of basaloid and ductal cells. ${ }^{1}$ Immunohistochemistry can help distinguish HMSC from HPV-related basaloid SCC, as the latter lacks the myoepithelial cells (often positive for cytokeratin, S100, actin, calponin, and p63) that make up salivary gland neoplasms. ${ }^{13}$

From a molecular standpoint, the case we report herein is unique due to its association with high-risk HPV type 16, which, to the best of our knowledge, has only been reported twice previously, and in neither of these reports were the clinical outcomes specifically highlighted. ${ }^{3,6}$ Most HPV-positive head and neck carcinomas arise from the oropharynx, with the sinonasal tract now recognized as another anatomic "hotspot" for HPV-related neoplasms. ${ }^{3,10,14}$ Sinonasal tract HMSC is most often associated with HPV type $33 .{ }^{4}$ Here, we describe the case of an HMSC with HPV-16 identified by both RT-PCR (reverse transcription polymerase chain reaction) and HPV-16/18 RNA in situ hybridization associated with an aggressive clinical course. Although anecdotal, the relationship between HPV subtype and clinical behavior in HMSC is the one that needs to be better delineated, as it may have important implications for patient stratification.

\section{Conclusion}

Here, we present an HPV16-associated case of HMSC with early and progressive metastatic disease. As more HMSC cases are identified, the clinical management of this entity may be better defined. We propose that HPV subtype may have prognostic importance and that the relationship between subtype and clinical behavior warrants further investigation.

\section{Presentation}

This case was presented at the North American Skull Base Society Annual Meeting in February 2019, Orlando, Florida, United States.

\section{Funding}

The author(s) received no financial support related to this manuscript.

\section{Conflicts of Interest}

All authors have no potential conflicts of interest to declare.

\section{References}

1 Hwang SJ, Ok S, Lee HM, Lee E, Park IH. Human papillomavirusrelated carcinoma with adenoid cystic-like features of the inferior turbinate: a case report. Auris Nasus Larynx 2015;42(01): 53-55

2 Rooper LM, McCuiston AM, Westra WH, Bishop JA. SOX10 immunoexpression in basaloid squamous cell carcinomas: a diagnostic pitfall for ruling out salivary differentiation. Head Neck Pathol 2018 (e-pub ahead of print). doi:10.1007/s12105018-0990-7

3 Bishop JA, Andreasen S, Hang JF, et al. HPV-related multiphenotypic sinonasal carcinoma: an expanded series of 49 cases of the tumor formerly known as HPV-related carcinoma with adenoid cystic carcinoma-like features. Am J Surg Pathol 2017;41(12): 1690-1701

4 Bishop JA, Ogawa T, Stelow EB, et al. Human papillomavirusrelated carcinoma with adenoid cystic-like features: a peculiar variant of head and neck cancer restricted to the sinonasal tract. Am J Surg Pathol 2013;37(06):836-844

5 Thompson LDR, Franchi A. New tumor entities in the 4th edition of the World Health Organization classification of head and neck tumors: nasal cavity, paranasal sinuses and skull base. Virchows Arch 2018;472(03):315-330

6 Hang JF, Hsieh MS, Li WY, et al. Human papillomavirus-related carcinoma with adenoid cystic-like features: a series of five cases expanding the pathological spectrum. Histopathology 2017;71 (06):887-896

7 Alos L, Moyano S, Nadal A, et al. Human papillomaviruses are identified in a subgroup of sinonasal squamous cell carcinomas with favorable outcome. Cancer 2009;115(12):2701-2709

8 Andreasen S, Bishop JA, Hansen TV, et al. Human papillomavirusrelated carcinoma with adenoid cystic-like features of the sinonasal tract: clinical and morphological characterization of six new cases. Histopathology 2017;70(06):880-888

9 Chouake RJ, Cohen M, Iloreta A-M. Case report: HPV-related carcinoma with adenoid cystic-like features of the sinonasal tract. Laryngoscope 2018;128(07):1515-1517

10 Adamane SA, Mittal N, Teni T, Pawar S, Waghole R, Bal M. Human papillomavirus-related multiphenotypic sinonasal carcinoma with unique HPV type 52 association: a case report with review of literature. Head Neck Pathol 2018 (e-pub ahead of print). doi:10.1007/s12105-018-0969-4

11 Shah AA, Lamarre ED, Bishop JA. Human papillomavirus-related multiphenotypic sinonasal carcinoma: a case report documenting the potential for very late tumor recurrence. Head Neck Pathol 2018;12(04):623-628

12 Hung Y, Chung YY, Chen ML, Guo JY. HPV-related sinonasal carcinoma with adenoid cystic-like features with intracranial invasion. Ear Nose Throat J 2018;97(07):191-192

13 Bishop JA, Westra WH. Human papillomavirus-related multiphenotypic sinonasal carcinoma: an emerging tumor type with a unique microscopic appearance and a paradoxical clinical behaviour. Oral Oncol 2018;87(87):17-20

14 Lewis JS Jr, Westra WH, Thompson LDR, et al. The sinonasal tract: another potential "hot spot" for carcinomas with transcriptionally-active human papillomavirus. Head Neck Pathol 2014;8(03):241-249 